\title{
Wer hat die bessere Moral?
}

Reiche lügen und betrügen häufiger als Menschen mit niedrigerem sozialem Rang. Außerdem verhalten sie sich im Straßenverkehr rücksichtsloser als die sozial schlechter Gestellten. Dies ergaben sieben Experimente von amerikanischen und kanadischen Forschern.

- Für die ersten beiden Experimente wurde das Verhalten von Verkehrsteilnehmern an Kreuzungen und Fußgängerübergängen beobachtet. Wie sich zeigte, sind es überwiegend die Lenker teurer Fahrzeuge, die an Kreuzungen die Vorfahrt missachten und die an Zebrastreifen nicht für wartende Fußgänger halten.

Im dritten Experiment wurde der soziale Status der Probanden mittels Selbstauskunft ermittelt. Anschließend wurden den Versuchsteilnehmer acht Szenarien

vorgespielt, in denen sich ein Schauspieler unethisch verhielt. Die Versuchspersonen sollten dann angeben, ob sie sich in einer vergleichbaren Situation ähnlich verhalten würden wie der Schauspieler. Auch hier neigten eher die sozial besser Gestellten zu einem verwerflichen Verhalten.

Weitere Tests zeigten, dass die sozial besser Gestellten eher beim Spiel um Geld betrogen, Bewerber bei einem fik-

\section{Kommentar}

Auf den ersten Blick ist das Resultat verwunderlich, sind es doch eher die unteren Schichten, die es eigentlich nötiger hätten, zu schummeln und sich einen Vorteil zu verschaffen. Aber nicht der höhere soziale Rang per se ist verantwortlich für das weniger moralische Verhalten, sondern vielmehr die Einstellung der Probanden zur Gier: Angehörige höherer Schichten sahen diese Charaktereigenschaft als weniger negativ an. Auch arbeiteten sie oft in Beru- tiven Vorstellungsgespräch belogen oder zu viel erhaltenes Wechselgeld behielten.

- P. K. Piff et al.

(Department of Psychology, University of California, Berkeley, C4 94720, USA E-mail: paulpiff@gmail.com.) Higher social class predicts increased unethical behavior. Published online on 27 February 2012 in Proc Natl Acad Sci.; doi:

\section{Verheiratete überleben Herzoperationen besser}

\begin{abstract}
Wie verschiedene Studien gezeigt haben, leben Verheiratete länger. Dies gilt sowohl für die Gesamtpopulation als auch in klinischen Studien nach medizinischen Eingriffen.
\end{abstract}

- In einer regionalen Probe $(n=569)$ verglichen Wissenschaftler der Emory University in Atlanta (Georgia) mittels Krankenblättern und psychosozialen Interviews die 5-Jahresmortalität nach Herzoperationen von verheirateten Personen mit der von verwitweten, geschiedenen und nicht verheirateten Personen.

Nach Wegrechnen der demografischen, prä- und postchirurgischen Faktoren hatten die unverheirateten Personen ein 1,90-fach höheres Mortalitätsrisiko. Männer und Frauen unterschieden sich nicht. Unmittelbar nach der
Operation war diese Differenz auf das 3,33-fache erhöht. Die Langzeitmortalität war bei den nicht Verheirateten um den Faktor 1,71 erhöht.

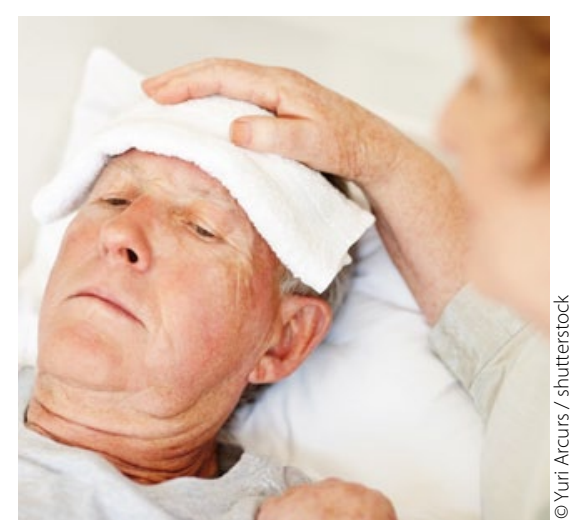

Ein Ehepartner hilft, kritische Situationen zu überstehen.

\begin{abstract}
- E. L. Idler et al.
Mending broken hearts. Marriage and survival following cardiac surgery. J Health Soc Behavior 2012; 53: 33-49; doi: $10.1177 / 0022146511432342$
\end{abstract}

\section{Kommentar}

Verheiratete Patienten sind vor einer Operation deutlich optimistischer und haben weniger Angst vor Schmerzen als Alleinstehende. Sie haben deshalb in den kritischen Monaten nach einer Herzoperation höhere Überlebenschancen als die unverheirateten Personen. Die Autoren der Studie führen das niedrigere Mortalitätsrisiko unter anderem auch darauf zurück, dass Eheleute den Lebenswandel ihres kranken Partners überwachten und mit dazu beitrugen, Risikofaktoren auszuschlieBen.

K. MALBERG = 